\title{
FRECUENCIA DEANTICUERPOS CONTRA Neospora caninum Y Toxoplasma gondii EN CANES CON SIGNOS CLÍNICOS DE AFECCIÓN NEUROMUSCULAR
}

\author{
Frequency of Antibodies Against Neospora caninum y Toxoplasma gondil in \\ Dogs With Clinical Signs of Neuromuscular Disease
}

\author{
Nelson Ruíz R. ${ }^{1}$, Eva Casas A. ${ }^{1,4}$, Francisco Suárez A. ${ }^{2}$, Diego Díaz C. ${ }^{3}$, \\ Viviana Fernández $\mathbf{P}^{3}$
}

\section{RESUMEN}

Toxoplasma gondii y Neospora caninum son causantes de enfermedades neuromusculares en canes. El objetivo de este estudio de Caso-Control fue encontrar la asociación entre la presencia de anticuerpos contra $N$. caninum y T. gondii en canes con signos clínicos neuromusculares. Se colectó muestras de sangre a 96 y 120 canes con y sin signos clínicos de afección neuromuscular, respectivamente. Los sueros se analizaron con la prueba de Inmunofluorescencia Indirecta (IFI) para la detección de anticuerpos (IgG), donde los sueros con fluorescencia completa del taquizoíto en la dilución 1:50 fueron considerados positivos. La frecuencia para T. gondii fue de $24.0 \pm 8.5 \%$ (23/96) y de $3.3 \pm 3.1$ (4/120) para canes con y sin afecciones neuromusculares, respectivamente, y para $N$. caninum fue de $5.2 \pm 4.4(5 / 96)$ y de $1.7 \pm 2.5(2 / 120)$ para canes con y sin afecciones neuromusculares, respectivamente. Se observó asociación significativa entre la afección neuromuscular y la presencia de anticuerpos anti-T. gondii, mas no así con anticuerpos anti- $N$. caninum.

Palabras clave: Toxoplasma gondii, Neospora caninum, canes, afección neuromuscular, inmunofluorescencia indirecta, anticuerpos IgG

\section{Abstract}

Toxoplasma gondii and Neospora caninum can cause neuromuscular disorders in dogs. The aim of the Case-Control study was to determine the association between antibodies anti $N$. caninum and anti-T. gondii in dogs with neuromuscular disorders. Blood samples were collected in 96 and 120 dogs with and without clinical signs of neuromuscular disorders respectively. Serum samples were analyzed by the indirect immunofluorescence test (IFI) for the detection of IgG antibodies, considering positive the sera showing complete fluorescence of the tachyzoite in the dilution 1:50. The

\footnotetext{
${ }^{1}$ Laboratorio de Microbiología y Parasitología Veterinaria, ${ }^{2}$ Laboratorio de Medicina Veterinaria Preventiva, ${ }^{3}$ Clínica de Animales Menores, Facultad de Medicina Veterinaria, Universidad Nacional Mayor de San Marcos, Lima

${ }^{4}$ E-mail: evacasas@gmail.com
} 
frequency for $T$. gondii was $24.0 \pm 8.5 \%$ (23/96) and $3.3 \pm 3.1$ (4/120) for dogs with and without signs of neuromuscular disorders, and for $N$. caninum was $5.2 \pm 4.4$ (5/96) and 1.7 \pm 2.5 (2/120) for dogs with and without signs of neuromuscular disorders, respectively. There was a significant association between neuromuscular disorders and the presence of anti-T. gondii antibodies but not in the case of the presence of anti- $N$. caninum antibodies.

Key words: Toxoplasma gondii, Neospora caninum, dogs, neuromuscular disease, indirect immunofluorescence, IgG antibodies

\section{INTRODUCCIÓN}

Toxoplasma gondii y Neospora caninum son protozoarios apicomplexos con ciclos biológicos heteroxenos que tienen una distribución mundial y afectan a una gran variedad de especies animales. Los sistemas gastrointestinal, respiratorio y neuromuscular son afectados en los canes. Las manifestaciones clínicas por problemas neuromusculares son similares por lo que, inicialmente, las casos producidos por Neospora caninum fueron confundidas con toxoplasmosis. No obstante, aunque estas enfermedades son similares, la toxoplasmosis parece ser más frecuente en gatos y la neosporosis en canes (Dubey y Lappin, 1998).

Ambos agentes parasitarios tienen un ciclo de vida del tipo predador (hospedero definitivo) - presa (hospedero intermediario). La reproducción sexual ocurre en el predador y la reproducción asexual en la presa (Rojas, 2003). Los hospederos definitivos de $N$. caninum son cánidos como el perro doméstico (Lindsay et al., 1999) y el coyote (Gondim et al., 2004), mientras que los hospederos definitivos de T. gondii son el gato (Dubey et al., 1970) y otros felinos (Jewell et al., 1972). Además, los canes pueden actuar como hospederos intermediarios y definitivos para $N$. caninum (Dubey, 2003) y como hospederos intermediarios para T. gondii (Lindsay et al., 1996).
Ambos protozoarios tienen tres estadios infecciosos: los esporozoítos en ooquistes esporulados, los taquizoítos (de multiplicación rápida) y los bradizoítos (de multiplicación lenta) en quistes tisulares. Los ooquistes son excretados con las heces, mientras que los taquizoítos y bradizoítos se encuentran en los tejidos de los hospederos intermediarios (Dubey y Lappin, 1998).

Existen diversas clasificaciones para los signos clínicos de la toxoplasmosis canina, dependiendo de su localización en los sistemas respiratorio, neuromuscular, gastrointestinal, o en una infección generalizada (Dubey y Lappin, 1998; Rojas, 2003). En la neosporosis canina, los signos clínicos son similares a los de la toxoplasmosis canina, predominando los problemas neurológicos y anormalidades musculares.

En el Perú, se han realizado estudios sobre la presencia de $N$. caninum en canes, reportándose una seroprevalencia de $29 \%$ en Chachapoyas (Horna et al., 2003), y de $19 \mathrm{y}$ $33 \%$ en canes de establos lecheros en el Valle del Mantaro (Cornejo et al., 2004) y de Lima (Del Campo et al., 2003). Asimismo, investigaciones sobre la presencia de $T$. gondii en el país han sido realizadas en diversas especies de animales domésticos y silvestres como cabras, ovejas, cerdos, monos, alpacas, llamas y vicuñas, habiéndose reportado recientemente una frecuencia de $36.5 \%$ de $T$. gondii en canes de Lima (Morales et al., 2008). 
En ese sentido, el objetivo del presente estudio fue investigar acerca de la presencia de anticuerpos IgG anti- $T$. gondii y anti- $N$. caninum en canes con afección neuromuscular, así como evaluar el tipo de asociación existente entre la presencia de afección neuromuscular y la presencia de anticuerpos IgG, proporcionando de esta manera un mayor sustento a la decisión del clínico veterinario en la ampliación de las posibilidades del diagnóstico diferencial para afecciones neuromusculares.

\section{Materiales y Métodos}

Se realizó un estudio de Caso-Control. Como Caso se consideró a canes de dos o más meses de edad con dos o más signos clínicos neuromusculares, y como Control a canes de dos o más meses de edad sin signos o con un solo signo clínico de afección neuromuscular. Se consideró como afección neuromuscular los signos clínicos de mialgia, nistagmo, ataxia, paresia, parálisis y convulsiones.

Se colectaron muestras de sangre de 96 canes del grupo Casos y de 120 canes del grupo Control en la Clínica de Animales Menores de la Facultad de Medicina Veterinaria de la Universidad Nacional Mayor de San Marcos (FMV-UNMSM), Lima, durante un periodo de dos años (agosto de 2006 a julio de 2008). Las muestras se colectaron indistintamente de la raza o sexo del animal. El suero se separó mediante centrifugación y fue almacenado a $-20{ }^{\circ} \mathrm{C}$ hasta su procesamiento con la prueba de Inmunofluorescencia Indirecta (IFI) en el Laboratorio de Parasitología de la FMV-UNMSM.

La prueba de IFI se realizó utilizando un conjugado canino del laboratorio VMRD (Pullman, WA-USA), siguiendo el protocolo estándar (Sánchez et al., 2002). La muestra se consideró positiva al observarse fluorescencia completa del taquizoíto, y negativa cuando ocurrió fluorescencia parcial (apical) o ausente.
Se calculó la frecuencia relativa de anticuerpos así como el respectivo intervalo de confianza para cada protozoario. Asimismo, se evaluó la asociación entre la seropositividad y el estado del animal (afección neuromuscular) mediante el cálculo del Odds Ratio para cada protozoario.

\section{Resultados}

La frecuencia de canes seropositivos a T. gondii y con afecciones neuromusculares fue de $24.0 \pm 8.5 \%$ (23/96), y en canes sin afección neuromuscular fue de $3.3 \pm 3.1 \%$ (4/120). Asimismo, en el caso de N. caninum, la seroprevalencia fue $5.2 \pm 4.4 \%(5 / 96)$ y de $1.7 \pm 2.5 \%(2 / 120)$ en canes con y sin afección neuromuscular, respectivamente (Cuadro 1).

Se encontró una asociación significativa entre la presencia de afección neuromuscular y presencia de anticuerpos anti-T. gondii, siendo el Odds Ratio de 9.1 con un intervalo de confianza entre 3.0 y 27.4. Asimismo, la asociación entre la presencia de afección neuromuscular y la presencia de anticuerpos anti- $N$. caninum resultó en un Odds Ratio de 3.24 con intervalo de confianza entre 0.61 y 17.29 , indicando independencia entre ambas variables.

Adicionalmente, se observó que mialgia y ataxia fueron los signos clínicos más frecuentes en los animales seropositivos a $T$. gondii y a $N$. caninum, y nistagmo en menor nivel.

\section{Discusión}

La frecuencia de IgG anti-T. gondii en canes con signos de afección neuromuscular fue de $24.0 \pm 8.5 \%$, valor estadísticamente similar a las frecuencias halladas en Brasil (Brito et al., 2002) y Argentina (Locatelli et al., 2006; Venturini et al., 2007) en canes con similares signos clínicos; sin embargo, no es 
Cuadro 1. Frecuencia de Toxoplasma gondii y Neospora caninum en canes según la presencia de afecciones neuromusculares (Lima, 2006-2008)

\begin{tabular}{lcccc}
\hline \multirow{2}{*}{ Protozoario } & Afección & Canes & \multicolumn{2}{c}{ Canes positivos } \\
\cline { 4 - 5 } T. gondii & No & 120 & 4 & $\% \pm$ I.C. $^{1}$ \\
\cline { 3 - 5 } N. caninum & Sí & 96 & 23 & $3.3 \pm 3.1$ \\
& No & 120 & 2 & $24.0 \pm 8.5$ \\
& Sí & 96 & 5 & $5.7 \pm 2.5$ \\
\hline
\end{tabular}

${ }^{1}$ Intervalo de confianza del $95 \%$

posible asegurar que cada caso haya sido debido a una toxoplasmosis clínica, dada la posibilidad de que los signos neurológicos sean originados por otras infecciones (Brito et al., 2002). No obstante, se ha planteado que la magnitud de los títulos de anticuerpos en los canes es indicadora de enfermedad clínica (Locatelli et al., 2006).

La frecuencia de IgG anti-T. gondii en canes sin manifestaciones neuromusculares (3.3 $\pm 3.1 \%)$ se encontró por debajo de frecuencias de 25 a $85 \%$ reportadas en otros países en canes sin signos clínicos neuromusculares (García et al., 1999; Aricapa et al., 2003; Barbosa et al., 2003; Sedlak y Bartova, 2006); sin embargo, esto depende largamente de la distribución del parásito, ya que también hay reportes con frecuencias similares a las del presente estudio (Lin, 1998). Recientemente, se ha reportado una frecuencia de $36.5 \%$ de $T$. gondii en canes sin signos clínicos de afección neuromuscular en varios distritos de Lima (Morales et al., 2008). Es posible que la baja frecuencia pueda deberse a que la gran mayoría de los canes eran mascotas mantenidas dentro del hogar, con menor probabilidad de tener contacto con roedores y palomas, además de ser alimentadas con concentrado (De Souza et al., 2003).

La frecuencia de IgG anti- $N$. caninum en canes con signos de afección neuromus- cular fue de $5.2 \pm 4.4 \%$, similar a las frecuencias obtenidas en otros estudios en canes con signos neurológicos (Cheadle et al., 1999; Mineo et al., 2001; Varandas et al., 2001), aunque también se han reportado valores inferiores (Locatelli et al., 2006; Venturini et al., 2007).

Por otro lado, la frecuencia encontrada en canes sin manifestaciones neuromusculares $(1.7 \pm 2.5 \%)$ fue similar a otros reportes (Sawada et al., 1998; Wouda et al., 1999) en canes mantenidos dentro de casas en zonas urbanas. Las diferencias entre frecuencias de seropositividad entre canes mantenidos dentro y fuera del hogar se deben a que estos últimos tienen mayor probabilidad de contacto con otras especies infectadas (felinos, roedores y cánidos silvestres) y los canes de establos tienen un estrecho contacto con bovinos (Moore, 2005). Es así, que en canes de establos lecheros de la cuenca izquierda del valle del Mantaro se encontró una seroprevalencia de $19.4 \pm 7 \%$ (Cornejo et al., 2004) y en canes de establos lecheros del valle de Lima de $32.7 \pm 9 \%$ (Del Campo et al., 2003).

Los signos clínicos más frecuentes en animales con afección neuromuscular que resultaron positivos a la prueba de IFI fueron mialgia y ataxia. La mialgia podría deberse a la multiplicación excesiva de los protozoarios en las células musculares, causando inflama- 
ción y dolor. Por otro lado, la ataxia se debe principalmente a una lesión a nivel del sistema nervioso central y es mencionada como signo clínico frecuente en casos de toxoplasmosis y neosporosis canina (Mineo et al., 2001; Brito et al., 2002; Locatelli et al., 2006).

La asociación significativa entre la presencia de IgG anti-T. gondii y afección neuromuscular no concuerda con el reporte de Varandas et al. (2001); sin embargo, esta asociación está fuertemente ligada a la presencia de otros factores que causen signos neuromusculares. La ocurrencia de determinados signos clínicos depende principalmente de la localización del parásito en el organismo hospedero. La afección neuromuscular es una característica común entre ambas infecciones protozoarias (Dubey y Lappin, 1998), dada su predilección por los sistemas nervioso y muscular. Dolor muscular (Barber, 1998; Pereira y Pérez, 2002), nistagmo (Barber, 1998; Tarlow et al., 2005), ataxia (Barber, 1998; Dubey y Lappin, 1998), paresia, paraparesia, parálisis (Dubey y Lapin, 1998; Georgieva et al., 2006) y convulsiones (Alves y De Lima, 2004; Tarlow et al., 2005) representan los principales signos clínicos de enfermedad neuromuscular descritos para Toxoplasma gondii y Neospora caninum.

En el presente estudio se utilizó la prueba de Inmunofluorescencia Indirecta para el diagnóstico serológico por ser una de las más utilizadas para detectar anticuerpos anti- $T$. gondii y anti- $N$. caninum en canes (Dubey y Beattie, 1988; Dubey et al., 2007). La prueba brinda resultados comparables a los obtenidos por el Dye Test y se le considera más segura ya que no utiliza taquizoítos vivos. Se reporta una sensibilidad y especificidad para T. gondii de 97.3 y $96 \%$, respectivamente (Gharavi et al., 2008). Asimismo, la prueba se puede adaptar para detectar IgG, IgM o IgA (Dubey y Lappin, 1998). En este estudio se utilizó para la detección de anticuerpos IgG, ya que el objetivo fue determinar la frecuencia de serorreactores, mas no la frecuencia de infección aguda en animales con signos clínicos de enfermedad neuromuscular.

\section{Conclusiones}

- $\quad$ El $24.0 \pm 8.5 \%$ (23/96) de animales con afección neuromuscular presentaron anticuerpos contra Toxoplasma gondii.

- Se observó asociación estadística significativa entre los reactores a T. gondii y la variable afección neuromuscular.

- $\quad$ El $5.2 \pm 4.4 \%(5 / 96)$ de animales con afección neuromuscular presentaron anticuerpos contra Neospora caninum.

- No se observó asociación estadística significativa entre los reactores a $N$. caninum y la variable afección neuromuscular.

\section{LITERATURA CitadA}

1. Alves T, De Lima R. 2004. Encephalitis caused by Neospora caninum and Toxoplasma gondii in dogs. Clínica Vet 48: 44-52.

2. Aricapa HJ, Pérez JE, Cardona JM, Piedrahita A. 2003. Seroprevalencia de toxoplasmosis humana y canina en el municipio de Manizales, año 2003. Biosalud 14: 9-17.

3. Barber J. 1998. Canine neosporosis. Waltham Focus 8(1): 25-29.

4. Barbosa MVF, Guimaraes JE, Almeida MAO, Gondim LFP, Regis GB. 2003. Frequency of IgG antibodies against - Toxoplasma gondii in sera of stray dogs in the city of Salvador - Bahia, Brazil. Braz J Vet Res Anim Sci 40: 457465.

5. Brito AF, Souza LC, Silva AV, Langoni H. 2002. Epidemiological and serological aspects in canine toxoplasmosis in animals with nervous symptoms. Mem Inst Oswaldo Cruz 97(1): 31-35.

6. Cornejo N, Chávez A, Casas E, Arana C. 2004. Seroprevalencia de Neospora caninum en perros de establos lecheros de la cuenca izquierda del valle del Mantaro. Rev Inv Vet, Perú 15(1): 70-75. 
7. Cheadle MA, Lindsay DS, Blagburn BL.1999. Prevalence of antibodies to Neospora caninum in dogs. Vet Parasitol 85: 325-330.

8. De Souza SLP, Gennari SM, Yai LEO, D'Auria SRN, Cardoso SMS, Guimaraes JS, Dubey JP. 2003. Ocurrence of Toxoplasma gondii antibodies in sera from dogs of the urban and rural areas from Brazil. Braz J Vet Parasitol 12(1): 1-3.

9. Del Campo J, Chávez A, Delgado A, Falcón, Casas E, Serrano E. 2003. Frecuencia de Neospora caninum en perros de establos lecheros del valle de Lima. Rev Inv Vet, Perú 14: 145-149.

10. Dubey JP, Miller NL, Frenkel JK. 1970. The Toxoplasma gondii oocyst from cat feces. J Exp Med 132: 636-662.

11. Dubey JP, Beattie CP. 1988. Toxoplasmosis of animals and man. Boca Raton, USA: CRC Press. 220 p.

12. Dubey JP, Lappin MR. 1998. Toxoplasmosis and neosporosis. In: Greene CE (eds). Infectious diseases of the dog and cat. $2^{\text {nd }}$ ed. Philadelphia: WB Saunders. p 493-509.

13. Dubey JP. 2003. Review of Neospora caninum and neosporosis in animals. Korean J Parasitol 41(1): 1-16.

14. Dubey JP, Schares G, Ortega-Mora LM. 2007. Epidemiology and control of neosporosis and Neospora caninum. Clin Microbiol Rev 20: 323-367.

15. Garcia JL, Navarro IT, Ogawa L, Oliveira RC. 1999. Seroepidemiology of toxoplasmosis in cats and dogs from rural properties of Jaguapita county, Paraná state, Brazil. Ciência Rural 29(1): 99-104.

16. Georgieva DA, Prelezov PN, Koinarski VTS. 2006. Neospora caninum and neosporosis in animals $-\mathrm{a}$ review. Bulg J Vet Med 9(1): 1-26.

17. Gharavi MJ, Oormazdi H, Roointan ES. 2008. A comparative study on sensitivity and specificity of conventional and unconventional IgG and IgM assays for diagnosis of toxoplasmosis. Iranian J Publ Heatlh 37(4): 42-45.
18. Gondim LFP, McAllister MM, Pitt WC, Zemlicka DE. 2004. Coyotes (Canis latrans) are definitive hosts of Neospora caninum. Int J Parasitol 34: 159-161.

19. Horna S, Chávez A, Casas E, Serrano E. 2003. Seroprevalencia de Neospora caninum en caninos en dos distritos de la provincia de Chachapoyas. Rev Inv Vet, Perú 14: 150-154.

20. Jewell ML, Frenkel JK, Johnson KM, Reed V, Ruiz A. 1972. Development of Toxoplasma oocysts in neotropical felidae. Am J Trop Med Hyg 21: 512-517.

21. Lin DS. 1998. Seroprevalences to Toxoplasma gondii in privately-owned dogs in Taiwan. Prev Vet Med 35: 21-27.

22. Lindsay DS, Dubey JP, Butler JM, Blagburn BL. 1996. Experimental tissue cyst induced Toxoplasma gondii infections in dogs. J Euk Microbiol 43(5): 113s (Abstr).

23. Lindsay DS, Upton SJ, Dubey JP. 1999. A structural study of the Neospora caninum oocyst. Int $\mathrm{J}$ Parasitol 29: 1521-1523.

24. Locatelli DR, Dal Pizzol J, FridlundPlugge N, Hoffmann DCS, Richartz RRTB, Patricio MAC, et al. 2006. Ocorrência de anticorpos anti-Neospora caninum e anti-Toxoplasma gondii em caes com sinais neurológicos atendidos no Hospital Veterinário da Universidade Federal do Paraná. En: XIV Congresso Brasileiro de Parasitologia Veterinária. Rio de Janeiro: Colégio Brasileiro de Parasitologia Veterinária.

25. Mineo TWP, Silva DAO, Costa GHN, Von Ancken ACB, Kasper LH, Souza MA, et al. 2001. Detection of IgG antobodies to Neospora caninum and Toxoplasma gondii in dogs examined in a veterinary hospital from Brazil. Vet Parasitol 98: 239-245.

26. Moore DP. 2005. Neosporosis in South America. Vet Parasitol 127: 87-97.

27. Morales J, Noé N, Falcón N, Chávez A. 2008. Presencia de gatos como factor de riesgo para la presentación de infecciones por Toxoplasma gondii en canes. Rev Inv Vet, Perú 20: 128-133. 
28. Pereira A, Pérez M. 2002. Toxoplasmosis. Offarm 21(4): 123-129.

29. Rojas M. 2003. Nosoparasitosis de perros y gatos peruanos. Lima: Martegraf. $83 \mathrm{p}$.

30. Sánchez EL, Náquira CG, Vega ES. 2002. Manual de procedimientos para el diagnóstico serológico de las zoonosis parasitarias. Serie de Normas Técnicas $\mathrm{N}^{\circ}$ 32. Instituto Nacional de Salud. $57 \mathrm{p}$.

31. Sawada M, Park Ch, Kondo H, Morita T, Shimada A, Yamane I, Umemura T. 1998. Serological survey of antibody to Neospora caninum in japanese dogs. J Vet Med Sci 60: 853-854.

32. Sedlak K, Bartova E. 2006. The prevalence of Toxoplasma gondii IgM and IgG antibodies in dogs and cats from the Czech Republic. Vet Med-Czech 51: 555-558.

33. Tarlow JM, Rudloff E, Lichtenberger $M$, Kirby R. 2005. Emergency presentations of 4 dogs with suspected neurologic toxoplasmosis. J Vet Emerg Crit Care 15: 119-127.
34. Varandas NP, Rached PA, Costa GHN, Souza LM de, Castagnoli KC, Costa AJ da. 2001. Frequency of antibodies for Neospora caninum and Toxoplasma gondii in dogs in northest of Sao Paolo State. Sci Agrarias, Londrina 22(1): 105-111.

35. Venturini MC, Unzaga J, Basso W, Bacigalupe D, Larsen A, Pardini L, et al. 2007. Neosporosis y toxoplasmosis en perros con signos clínicos en diez años de diagnóstico serológico. En: XVII Reunión Científica Técnica de la Asociación Argentina de Veterinarios de Laboratorios de Diagnóstico (II). Santa Fe: Red de Helmintología para América Latina y el Caribe.

36. Wouda W, Dijkstra T, Kramer AM, Van Maanen C, Brinkhof JM. 1999. Seroepidemiological evidence for a relationship between Neospora caninum infections in dogs and cattle. Int J Parasitol 29: 1677-1682. 